\title{
Clinical case discussion: anesthesia for Cesarean section in a parturient with a large intrathoracic tumour
}

\author{
[Exposé clinique : l'anesthésie pour une césarienne chez une parturiente qui \\ présente une grosse tumeur intrathoracique]
}

Edward Crosby MD

Purpose: To report the anesthetic management of a parturient with a large intrathoracic tumour, presenting for Cesarean section.

Clinical features: A 28-yr-old parturient, gravida I, presented at 33 weeks gestation with a one month history of increasing cough and dyspnea. A computed tomography scan demonstrated a large mass filling the right hemothorax, causing mediastinal displacement to the left and carinal compression. Both mainstem bronchi were compressed and there was near total obliteration of the lumens of the right lobar bronchi. A decision was taken to expedite delivery to allow for staging and treatment of her disease and Cesarean section was scheduled. She was seen in consultation and prescribed oxygen by nasal prongs, dextromethorphan for cough and ranitidine the evening before and the morning of surgery. A subarachnoid block was performed and a block to the upper thoracic dermatomes was achieved; surgery proceeded uneventfully with the patient's head and upper body elevated about $15^{\circ}$ from the supine. The patient was discharged to the medical oncology service for evaluation and treatment.

Conclusions: Intrathoracic tumours are uncommon in pregnancy. The physiological changes of pregnancy may mask not only the initial presentation but also, even advanced intrathoracic disease. Regional anesthesia is the anesthetic of choice and is rarely contraindicated by maternal condition.

Objectif : Présenter la démarche anesthésique choisie dans le cas d'une parturiente, atteinte d'une grosse tumeur intrathoracique, admise pour une césarienne.

Éléments cliniques : Une parturiente de 28 ans, primigeste, admise à 33 sem de grossesse, présentait une toux et une dyspnée croissantes depuis un mois. Un examen tomodensitométrique a révélé une grosse masse remplissant l'hémothorax droit, causant un déplace- ment médiastinal vers la gauche et une compression de la carène. Les deux bronches principales étaient comprimées et il y avait une oblitération quasi totale des lumières bronchiques lobaires droites. On a décidé de hâter l'accouchement en procédant à une césarienne, ce qui a permis de déterminer le degré d'extension de la tumeur et son traitement. La patiente a reçu, en consultation, une prescription d'oxygène administré par lunettes nasales, de dextrométhorphane pour la toux et de ranitidine à prendre le soir et le matin précédant la césarienne. Un bloc sous-arachnoïdien a été réalisé jusqu'à atteindre les dermatomes thoraciques supérieurs; l'intervention chirurgicale s'est bien déroulée, la patiente étant en décubitus dorsal et ayant la tête et la partie supérieure du corps surélevées de $15^{\circ}$ environ. La patiente a reçu son congé et a été dirigée vers le service d'oncologie médicale pour évaluation et traitement de la tumeur.

Conclusion : Les tumeurs intrathoraciques sont rares pendant la grossesse. Les changements physiologiques de la grossesse peuvent masquer non seulement l'aspect initial mais aussi bien la maladie intrathoracique avancée. L'anesthésie régionale constitue le meilleur choix et est rarement contre-indiqué par l'état de la mère.

Case

A 28-yr-old parturient, G1, presented to our institution at 33 weeks gestation with a large intrathoracic tumour occupying the bulk of her right hemithorax and involving mediastinal structures. She had a history of recurrent bronchitis dating from childhood and experienced several exacerbations annually. Although she had smoked cigarettes in the past, she had not done so for about one year. She had been seen by her family doctor about one month earlier with complaints of cough,

From the Department of Anesthesiology, University of Ottawa, Ottawa, Ontario, Canada.

Address correspondence to: Dr. Edward Crosby, Room 2600, Tower 3, Ottawa Hospital, General Site, 501 Smyth Road, Ottawa,

Ontario KlH 8L6, Canada. Phone: 613-737-8187; Fax: 613-737-8189; E-mail: ecrosby@ottawahospital.on.ca

Accepted for publication September 19, 2000.

Revision accepted February 12, 2001. 


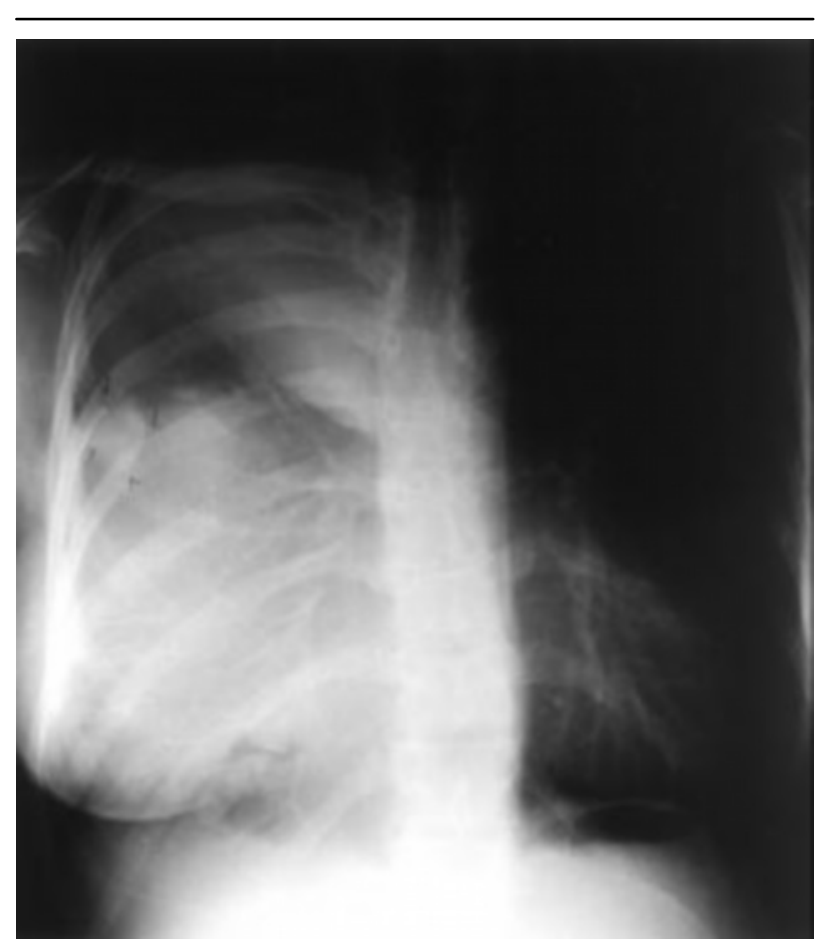

FIGURE 1 Chest $x$-ray demonstrating large right intrathoracic tumour, involving the middle and lower lobes. Metastatic nodules are noted in the right upper lobe and in the left lung.

fatigue, and dyspnea on minimal exertion. She was prescribed oral antibiotics and inhaled bronchodilators. Over the course of the subsequent month, her symptoms persisted and gradually worsened and she was briefly admitted to hospital for a course of $i v$ antibiotics. The results of pulmonary function tests done at that time were characteristic of obstructive airways disease. Five days before admission to our hospital, a chest $x$-ray demonstrated extensive consolidation of the right upper and middle lobes with little residual airspace in the right hemithorax (Figure 1). She was referred to our centre for assessment and management.

Following admission, a fine needle biopsy was done which was suggestive of sarcoma but inconclusive. A decision was taken to refer to the perinatology service in order to expedite delivery to allow for staging and treatment of her disease. She was seen by the high-risk obstetrical service and booked for Cesarean section the following day. An obstetrical anesthesia consultation was sought by the consultant perinatologist.

During consultation she complained of fatigue and dyspnea, although she did not appear distressed. She admitted to having slept fitfully for many days, her sleep being persistently interrupted by cough. She

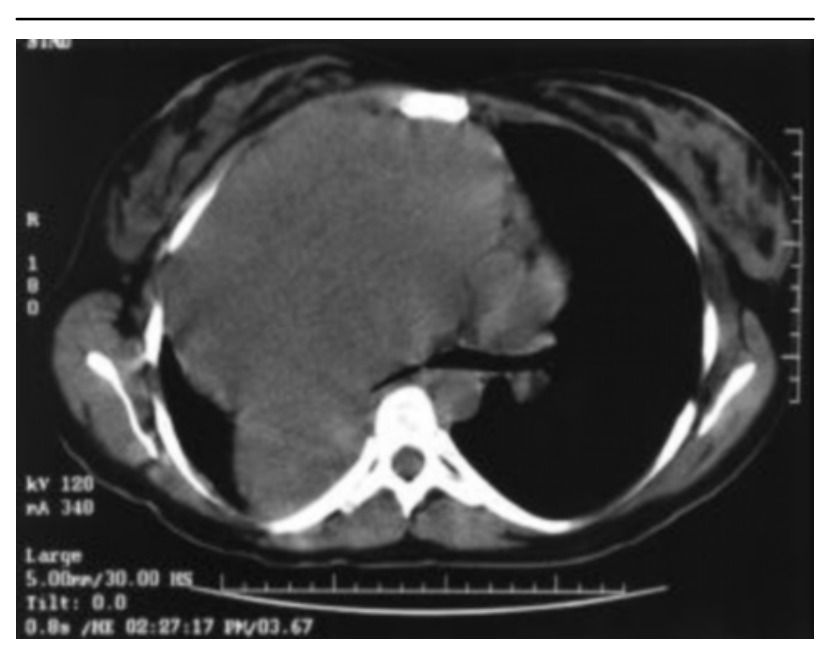

FIGURE 2 Computed tomography (CT) scan demonstrating carinal encasement and compression by the tumour with near obliteration of the right main stem bronchus. A moderate pneumothorax, resulting from the diagnostic needle biopsy is evident in the dorsolateral hemithorax.

complained of chest and upper abdominal discomfort, primarily left sided and dorsal, and noted that it was exacerbated by cough. As well, she complained of nasal congestion. Finally, she admitted to reflux and dysphagia, and stated that she was having trouble coordinating swallowing with her cough; she had lost $4 \mathrm{~kg}$ in the last ten days.

On examination, she was sitting at about 45 and was unable to lie at lesser degrees of head elevation without the onset of paroxysms of cough. Her respiratory rate was 24 and on room air her oxygen saturation was $95 \%$ by pulse oximetry. Despite her complaint of nasal congestion, there was no evidence suggestive of airway edema or superior vena cava syndrome (SVCS); the airway was adequate. Evaluation of the upper airway revealed no findings associated with difficult laryngoscopy and the trachea was midline. There was a split first heart sound and a soft systolic ejection murmur. The right hemithorax was dull to percussion, air entry was poor and there were bronchial breath sounds. Auscultation of the left lung was normal. The thoraco-lumbar spine was normal in appearance and on palpation.

She was anemic with a hemoglobin of $98 \mathrm{~g} \cdot \mathrm{L}^{-1}$; her electrolyte and coagulation screens were normal. A computed tomography (CT) with contrast was done and confirmed a large mass filling the right hemothorax, involving mediastinal elements and causing mediastinal displacement to the left; the carina was 
displaced posteriorly and compressed against the spine with narrowing of both mainstem bronchi, as well as near or total obliteration of the lumens of the right lobar bronchi. Both the SVC and the right pulmonary artery were encased and narrowed by tumour but neither was occluded. There was evidence of metastases to the left lung with multiple nodules being distributed throughout all lobes. A moderate right sided pneumothorax, attributable to the needle biopsy, was also noted (Figure 2). There was little evidence of aeration in the lung and it was felt that there was low potential for expansion of the pneumothorax.

She was prescribed oxygen by nasal prongs, dextromethorphan $15 \mathrm{mg}$ po every four hours as needed and ranitidine $150 \mathrm{mg}$ that evening and the following morning before surgery. Arterial blood gases were done with the patient breathing oxygen, $3 \mathrm{Lpm}$ by nasal prongs; the $\mathrm{pH}$ was $7.42, \mathrm{PaCO}_{2} 32 \mathrm{~mm} \mathrm{Hg}$, and $\mathrm{PaO}_{2} 112 \mathrm{mmHg}$. A regional anesthetic was recommended, specifically a subarachnoid block and the patient consented. The surgeon was advised of the likely requirement for some measure of head-up position during surgery.

Overnight, she experienced considerable symptomatic relief with the prescribed therapy. The following morning she was taken to the operating room; a large bore iv catheter was in situ in the left hand and was utilized for the surgery. After a $20 \mathrm{~mL} \cdot \mathrm{kg}^{-1}$ saline bolus $i v$, and $10 \mathrm{mg}$ of metoclopramide $i v$, a subarachnoid block was performed uneventfully, in the L3-4 interspace using a 27 GA non-cutting needle. Bupivacaine $0.75 \%$ in dextrose, $11.25 \mathrm{mg}$, was administered in combination with fentanyl $10 \mu \mathrm{g}$ and preservative-free morphine $100 \mu \mathrm{g}$. A bilateral block to the fourth thoracic dermatomes was achieved by briefly tipping the bed into a modest Trendelenburg while keeping the bed flexed at the hips; this brief manoeuver was well tolerated by the patient. Surgery proceeded uneventfully with the patient's head and upper body elevated about 15 from supine. A female infant in good condition was delivered and care was provided by an attending neonatologist. The infant required limited respiratory support in the neonatal intensive care for several days following delivery. This requirement was attributed to her premature status, and no evidence of neoplastic disease was evident in the infant. Further, there was no evidence of metastatic lesions on visual and later pathological inspection of the placenta. Two doses of fentanyl $25 \mu \mathrm{g} i$, were administered for cough control with good effect and an oxytocin infusion of $20 \mathrm{U} \cdot \mathrm{L}^{-1}$ was started following clamping of the umbilical cord. She was taken to the recovery room in good condition after a 40 -min pro- cedure. A total of $1500 \mathrm{~mL}$ of crystalloid was administered intra-operatively.

Three days postpartum she was transferred to the medical oncology service. Two days later she underwent an open lung biopsy under thoracic epidural anesthesia supplemented by small iv increments of fentanyl and midazolam. The procedure was well tolerated. The pathological diagnosis was that of Ewingtype sarcoma (primitive, undifferentiated small cell neoplasm).

\section{Discussion}

Maternal malignant lesions occur in only $0.1 \%$ of pregnancies and intrathoracic tumours are rarer still. ${ }^{1}$ Martin and Ferarri reported two parturients with large anterior mediastinal masses, both ultimately attributable to Hodgkin's disease. ${ }^{2,3}$ One patient was administered continuous spinal anesthesia for Cesarean delivery and the second received general anesthesia for tumour biopsy. Hodgkin's disease affects women predominantly in the childbearing years; its incidence has been reported as one in 6,000 pregnancies. Patients presenting in pregnancy with large intrathoracic masses, similar to those of Martin and Ferrari, are uncommon. Voluntary termination of the pregnancy is recommended to allow full evaluation of the abdomen and to avoid the teratogenic effects of both chemotherapy and ionizing radiation. Outcomes in pregnant patients are similar to those achieved by nonpregnant patients.

Primary cancers of the lung have surpassed breast cancer as the number one cause of death among women with cancer. ${ }^{l}$ Despite this statistic, lung cancer during pregnancy has been reported only a dozen times. ${ }^{1}$ Management of the cancer depends on the gestational age of the fetus, the clinical stage or operability of the tumour and whether or not the patient elects to continue the pregnancy. Surgical resection of operable tumours is the treatment of choice and is associated with the highest long-term maternal survival. Pregnancy does not appear to alter the course of the cancer, nor does the cancer adversely affect the fetus. Interestingly, the lack of adverse impact on the fetus occurs despite the fact that metastatic involvement of the placenta is common, occurring in two thirds of reported cases. ${ }^{l}$

Experience with chemotherapy during pregnancy has been predominantly with patients receiving treatment for breast cancer, Hodgkin's lymphoma, and leukemia. ${ }^{l}$ If the fetus is exposed beyond the period of organogenisis, neonatal outcome is usually good. The main risks to the fetus appear to those of growth retardation and preterm labour associated with poor nutri- 
tion, weight loss, and anemia.

The initial diagnosis of lung pathology in a parturient is complicated by the fact that the signs and symptoms that are consistent with normal pregnancy may be similar to those which herald the onset or presence of lung disease. Although most women complain of dyspnea by the middle of the third trimester, it begins in many during the first or second trimester. Dyspnea probably results from the parturient's subjective awareness of hyperventilation that is universally present in pregnancy. ${ }^{4}$ Fatigue and reduced exercise tolerance are also common complaints during pregnancy and are possibly due to increased body weight.

Three features help distinguish physiological (gestational) from pathological dyspnea. ${ }^{4,5}$ First, physiological dyspnea tends to onset earlier in pregnancy and often plateaus or improves as term approaches. Second, it is rarely extreme, and patients can usually maintain daily activities. Third, it is not associated with other respiratory symptoms such as cough. The dyspnea of pulmonary disorders is progressive, becoming most severe as gestation advances when the physiological loading is maximal. If dyspnea is extreme, has a limiting impact on normal activity, occurs at rest or with minimal exertion or is associated with a cough, maternal respiratory disease should be ruled out. ${ }^{4}$ Cough is not a normal finding in pregnancy and dyspnea that is acute in onset or progressive and intractable, especially if coupled with other signs and symptoms (i.e., orthopnea, paroxysmal nocturnal dyspnea), is also more likely to represent disease.

Even fairly severe intrathoracic disease may be masked by pregnancy. Jugular venous distention is seen in late pregnancy, as a result of the increase in circulating blood volume. ${ }^{4}$ However, right-sided pressures are not increased. Persistently or significantly elevated jugular venous pressure is a sign of elevated right atrial pressure and may reflect intrathoracic pathology. Pulmonary bibasilar crackles are occasionally present. ${ }^{4}$ These result from the atelectasis that occurs as the diaphragm moves cephalad compressing the lung bases and typically clear or are very much reduced following a deep inspiration.

Diagnosis in our patient may have been delayed by an early attribution of her symptoms to pregnancy rather than her tumour and a reluctance to subject her to diagnostic radiology because of the concerns regarding fetal exposure. The National Radiation Commission's (USA) recommendation for ionizing radiation exposure during pregnancy is to limit total maternal radiation throughout gestation to less than 5 rads (chest $x$-ray exposure 0.2 rads). An exposure of greater than 10 rads has been associated with signifi- cant fetal effects if administered during a sensitive gestational period. ${ }^{6}$ An exposure of $5-10$ rads is associated with a two-fold increase in the incidence of childhood leukemia.

There was also a reluctance to prescribe symptom relieving therapy (cough suppressant) because of the pregnancy despite the fact that the symptoms were disruptive and incapacitating and that the therapy was safe. Boyle suggests that the anatomical changes in chest wall configuration during pregnancy makes parturients more susceptible to chest wall injury, specifically rib fractures, than non-pregnant patients. ${ }^{7}$ The costal angles are widened in pregnancy and the lower ribs are pulled down with the cough. Tremendous intrapleural pressures are generated, which may result in rib fracture. This likely explains the chest discomfort and tenderness that our patient presented with and the tremendous relief obtained with cough suppression.

Maintenance of the patency of the tracheobronchial tree in a patient with a large intrathoracic mass involving the mediastinum may be dependent both on upright posture and preservation of normal intrathoracic dimensions. With a shift to the supine position, gravity becomes oriented across the anteroposterior dimensions of the chest cavity, potentially leading to collapse of the tracheo-bronchial tree as the mass compresses down upon it. The normal physiological changes of pregnancy may also interact with the pathological changes induced by large intrathoracic masses. The functional residual capacity decreases during pregnancy, approaching closing capacity, further reducing the lumenal size of the airways and also promoting collapse of some alveolar units not otherwise involved with tumour. ${ }^{5}$ Anesthesia in the supine patient also leads to a decrease in the dimensions of the rib cage with the loss of chest wall tone, a cephalad displacement of the dome of the diaphragm and a reduction in thoracic volume. The dimensions of the chest wall are decreased as lung volume moves to functional residual capacity and this loss of volume may further compromise the lumenal size of the compressed airways.

The supine position also causes an increase in central blood volume, which can further increase tumour blood volume and size. Edema, bleeding and hematoma formation in the tumour as a result of surgical biopsy can also further contribute to airway compromise. Although compression of the main pulmonary artery is relatively rare, due in part to the protective effect of the aorta, a higher pressure vessel, compression of either the pulmonary trunk or one of the main pulmonary arteries can result in sudden hypoxemia, hypotension or cardiac arrest. Patients 
with masses compressing the pulmonary artery may be relatively asymptomatic while awake yet may develop severe life-threatening hypoxemia, during anesthetic induction or under sedation. The assumption of the supine position, the change in chest wall muscle tone under general anesthesia and the institution of positive pressure ventilation all can combine to diminish pulmonary blood flow critically.

Parturients have increased metabolic requirements for oxygen and more rapidly become hypoxemic with apnea. This fact coupled with the increased potential for airway collapse in patients with large intrathoracic, mediastinal tumours both contraindicate the use of rapid-sequence induction of general anesthesia and discourage the use of elective general anesthesia.

SVCS caused by a mediastinal tumour has been reported with some frequency. ${ }^{1}$ It results from obstruction of venous drainage in the upper thorax. Increased venous pressure leads to: 1) dilatation of collateral veins of the upper part of the thorax and neck; 2) edema and plethora of the face, neck and upper torso; 3 ) suffusion and edema of the conjunctiva with or without proptosis; and 4) central nervous system (CNS) symptoms like headache, visual distortion or altered mentation. Venous distention is most prominent in the recumbent position but in most instances the veins do not collapse in the normal manner when the patient assumes the upright position. The most common symptom is shortness of breath with one of the earliest signs being facial or periorbital edema. CNS changes are characteristic of more rapidly progressive diseases. Cancer accounts for most cases of SVCS while benign causes are uncommon. ${ }^{1}$ Acute worsening of SVCS symptomatology has been reported to occur as a result of generous fluid administration and diuresis has been recommended in patients with SVCS causing obstructive symptoms, assuming that diuresis will also decrease tumour volume. One should bear in mind, however, that a diuresis may cause a decrease in preload leading to hypotension, worsening a situation already complicated by compromised venous return. Our patient did not have evidence of SVCS and no signs or symptoms were precipitated with perioperative fluid therapy although we were cognizant of the threat.

\section{Evaluation/preparation and care of the parturient with a large intrathoracic mass}

In addition to a thorough history and physical examination, recent posterior-anterior and lateral chest radiographs should be available. In general, the size of a mass and the degree of airway compression can accurately be established by $\mathrm{CT}$ scan. In addition to anatomic/radiographic studies of the chest, dynamic studies of the airway may be carried out in cooperative patients. Flow-volume loops may demonstrate the changes in flow rates at different lung volumes. Maximal inspiratory and expiratory flow-volume curves obtained with the patient in the upright and supine positions enable the functional degree of impairment to be quantified. There was such prominent evidence of severe airway narrowing and obliteration that we did not feel that dynamic assessments would add important clinical information in our patient. Flexible fibreoptic bronchoscopy under topical anesthesia is another method of evaluating dynamic airway obstruction. It allows assessment of the functional anatomy of the entire airway and the response of the airway to variations in intrathoracic pressure, particularly with changes from the supine to sitting and semi-prone positions. Finally, ultrasonography and echocardiography may be useful in assessing myocardial contractility and the degree of tumour encasement of the heart and great vessels.

Regional anesthesia was chosen to avoid instrumenting an obviously compressed and compromised airway. High thoracic spinal anesthesia may reduce expiratory forces and flows as the muscles of the abdominal wall are the principal expiratory muscles. A profound motor block impairs the expiratory force, especially in a parturient. ${ }^{2}$ However, it was our opinion that the potential problems surfacing during the operation, including maternal cough and positional dyspnea, would be manageable and preferable to the possible sequelae resulting from airway interventions. Subarachnoid block with a modest dose of hyperbaric bupivacaine was chosen over epidural block because of the increased certainty and density of the block and the ability to manipulate the block using gravity and the Valsalva manoeuver, if necessary. Anesthesia was supplemented with small increments of $i v$ fentanyl which were effective as cough suppressants.

We have presented the anesthetic and perioperative management of a young woman presenting in late gestation with a large intrathoracic tumour. Intrathoracic tumours are uncommon in pregnancy. The physiological changes of pregnancy may mask not only the initial presentation but also, even advanced intrathoracic disease. Regional anesthesia is the anesthetic of choice, is rarely contraindicated by the maternal condition, and was utilized successfully in this instance.

\section{Commentary}

Lesley-Ann Crone MD FRCPC

From the Department of Anesthesia, Royal University Hospital, University of Saskatchewan, Saskatoon, 
Saskatchewan, Canada.

This case report summarizes the complex perioperative considerations and anesthetic management of a parturient with a large intrathoracic tumour. Although the incidence of malignancy during pregnancy is presently rare, it is plausible that in the next few decades an increasing trend will emerge due to advances in assisted reproductive techniques. Case reports of childbirth after the age of 50 are no longer considered unusual and donor oocyte programs now enable postmenopausal women to conceive and deliver. ${ }^{8}$ Associated with advanced maternal age is an increased incidence of coexisting medical conditions including malignancy as well as physiologic changes of aging. 9

Crosby offers a thorough discussion of the physiologic changes occurring in pregnancy which can account for the chest discomfort experienced by this patient, but one would be remiss in attributing these complaints to only physiologic alterations. Metastatic disease leading to pathologic fractures in the vertebral column, ribs, chest wall and clavicle may be present, as well as intracranial lesions, depending on the primary tumour cell type. Preoperative metastatic evaluation including serum calcium, alkaline phosphatase, and liver function tests may be useful, but radiographic investigations may be limited due to the desire to limit fetal exposure. The presence of vertebral, spinal or intracranial metastases with mass effect will impact on the choice of anesthetic technique.

Pullerits' review of the anesthetic considerations for patients with mediastinal masses highlights three types of intrathoracic compromise. These are: 1) compression of the tracheobranchial tree; 2) compression of the pulmonary artery and heart; and 3) associated SVCS. ${ }^{10}$ The essential management strategies related to each of these problems, as outlined by Benumof, 1 may require certain alterations in relation to specific physiologic and anatomical alterations of pregnancy. It is usually preferable to irradiate the tumour if the cell type is sensitive, prior to biopsy and surgery to achieve some shrinkage of the tumour mass and alleviate symptomatology, but the benefits of this regime are often precluded due to the risks of ionizing radiation to the fetus. Interventions to relieve ventilatory obstruction include moving the patient to the lateral or the prone position. Left uterine displacement should always be attempted and is preferred to prevent aortocaval compression during pregnancy. However, achieving the prone position in an emergency scenario may be physically challenging as well as technically difficult, especially in the third trimester. Preoperative evaluation and preparation for such possibilities would be prudent. In this case report, the CT revealed that both the SVC and the right pulmonary artery were encased by tumour, again necessitating the importance of maintaining preload and the capability of changing the patient's position should sudden cardiovascular collapse occur. Left uterine displacement will assist the maintenance of preload. Although there was no evidence of SVCS, the use of lower extremity iv lines should be considered, and echocardiographic evaluation can add important information regarding the extent of tumour compression.

The author's choice of a regional technique rather than a general anesthetic is logical from the viewpoint of maintaining spontaneous ventilation, avoiding potentially lethal airway collapse reported in cases of mediastinal tumours, avoiding the potential complications of an existing pneumothorax. These risks coupled with the normal respiratory physiologic changes of pregnancy render these parturients at increased risk of morbidity and mortality ${ }^{12}$ during induction of general anesthesia. Intraspinal opioids (usually a single microdose) offers superior analgesia with a minimum side effect profile.

Many would argue against a subarachnoid block due to the unpredictable degree of hypotension and level of block, especially in a patient who did not tolerate recumbency. An epidural technique with incremental dosing through a catheter would allow for a more gradual onset block in this elective setting and potentially less cardiovascular instability. ${ }^{13}$ Emergency airway equipment, including a fibreoptic laryngoscope, rigid bronchoscope and high frequency jet ventilator, should be in the operating room. Although this surgery was being performed to expedite the oncological therapy in the mother and to remove the fetus from potentially lethal effects of possible radiotherapy and chemotherapy, and not as definitive tumour excision, it would be expeditious to have immediate access to cardiopulmonary bypass in the event of airway or cardiovascular collapse. An instrumental delivery of the fetus using either a vacuum extractor or forceps would be beneficial in avoiding increased maternal intra-abdominal and intrathoracic pressure and should be discussed with the obstetrical team prior to initiation of the surgery.

Such complex and rare cases as the one reported by Crosby, are best managed by a multidisciplinary team creating a periopertative plan.

\section{Commentary}

Beverly Morningstar MD FRCPC

From the Department of Anesthesia, Sunnybrook and Women's College Health Sciences Centre, University of Toronto, Toronto, Ontario, Canada. 
The case report and discussion by Crosby of a parturient with a large intrathoracic mass is noteworthy for several reasons. Most important is the reminder once again that significant pathology may be disguised by or dismissed as a variant of a healthy pregnancy. The literature abounds with examples of patients suffering delayed diagnosis and treatment for serious illnesses, especially malignancies, due either to symptoms confused with normal physiologic changes of pregnancy, or to a reluctance to perform necessary diagnostic tests. ${ }^{14-17}$ Of particular interest to anesthesiologists, the features that distinguish normal from pathological dyspnea in pregnancy are clearly summarized.

A review of guidelines for safe exposure to ionizing radiation during pregnancy warrants similar consideration. Contrary to the "knee-jerk" reaction to avoid radiation in pregnancy at any cost, we are reminded that pregnant women with fetal shielding can safely tolerate numerous chest $x$-rays when clinically indicated. One source suggests that the number of chest $x$ rays (two views) required for a cumulative 5 -rad dose, the maximum limit of ionizing radiation to which the fetus should be exposed, is more than $70,000 !^{18}$ Similarly, the number of CT scans of the chest (each using ten slices of $10-\mathrm{mm}$ thickness) required for a cumulative 5 -rad dose is greater than 50 examinations during pregnancy. ${ }^{18}$ Although there have been no documented adverse fetal effects reported, until more information is available, magnetic resonance imaging is arbitrarily not recommended for use in the first trimester. ${ }^{19}$ The risks to a pregnant woman's health caused by avoiding necessary diagnostic imaging almost always outweigh the minor hazards posed by low-dose radiation exposure of the developing infant. When diagnostic imaging is urgently required in the tenth through $17^{\text {th }}$ weeks of pregnancy, ultrasonography may present an alternative to ionizing radiation and is considered safe throughout pregnancy.

Case reports are a valuable addition to medical literature insofar as the reader may benefit from the author's experience when venturing into unfamiliar territory. With near-universal internet access, it is possible to become an expert on almost any uncommon condition with very little advance warning. Even a busy obstetric anesthesiologist working in a tertiary care centre has probably never encountered a parturient with a large intrathoracic mass. The rarity of a condition makes a case report all the more valuable if it presents a discussion of the full spectrum of anesthetic management. This should include guidelines for identifying those patients at greatest risk, and suggestions for both regional and general anesthesia.

Clearly, a local or regional anesthetic is the tech- nique of choice in any patient, pregnant or otherwise, with a large mediastinal mass. Crosby has thoroughly reviewed the respiratory and cardiovascular pathology, interactions with normal physiologic changes of pregnancy, and the worsening of these alterations induced by the supine position. Cough, the most disabling symptom preventing this patient from lying flat, was greatly improved with an antitussive. However, CT findings of carinal compression, narrowing of both main stem bronchi, and near total obliteration of the lumens of the right lobar bronchi (with an incidental finding of significant pneumothorax in the third trimester of pregnancy) suggest inevitable orthopnea, even after resolution of the cough.

In light of these clinical findings, it is curious that the author chose to manage this patient with a hyperbaric, subarachnoid block, based on the ability to manipulate the block using gravity. Reliance on a head-down position to ensure adequate anesthesia in a patient with a symptomatic mediastinal mass seems illogical. We are not told what position the patient was in during performance of the subarachnoid block; in a patient with orthopnea it is reasonable to assume that the sitting position was chosen. One of the common causes of failed spinal anesthesia is pooling of a hyperbaric drug, with lack of cephalad spread, associated with the use of the sitting position. ${ }^{20}$ Any delay in getting the patient into a comfortable supine position could possibly result in a level of anesthesia inadequate for Cesarean section. ${ }^{2} 1$

Hypotension is an added concern using a head-up position during spinal anesthesia. The resurgence in popularity of spinal anesthesia for elective Cesarean section notwithstanding, some anesthesiologists would still choose an epidural when it is desirable to avoid hypotension. ${ }^{22}$ Elevation of the legs does not reliably protect against a drop in blood pressure following spinal anesthesia for Cesarean section. ${ }^{23}$ Careful titration of incremental doses of epidural local anesthetic, along with a slower onset of anesthesia, permits better maternal cardiovascular compensation for sympathetic blockade. ${ }^{20}$ An epidural is much less gravity dependent and would allow the mother time to find a comfortable position on the operating room table. Another reason cited by the author for the choice of spinal rather than epidural anesthesia is the denser block. This patient had severe respiratory compromise; a dense motor block of the thoraco-abdominal segments could have prevented effective use of the accessory muscles of respiration and left the patient feeling anxious and even more dyspneic.

What happens when the obstetrician "nicks" the bowel or cuts a ureter? There is little evidence of planning for potential complications that prolong surgery 
beyond the expected duration of action of intrathecal bupivacaine in a patient who should not be subjected to general anesthesia. Even a combined spinal epidural, with an epidural ready for use should the need arise, is preferable to the finite duration of a spinal.

Patients may be relatively asymptomatic and yet have significant intrathoracic pathology that becomes life threatening upon induction of general anesthesia. A recent review of pre-anesthetic evaluation of children with anterior mediastinal masses ${ }^{24}$ suggests lower limits of two parameters which indicate when general anesthesia can be used safely. These are a tracheal area measured by CT scan of at least $50 \%$ of the area predicted for normal, and a peak expiratory flow rate of at least $50 \%$ of predicted. Goh et $a l^{2}{ }^{5}$ state that patients having more than $50 \%$ obstruction of the airway at the level of the lower trachea and main bronchi should have their femoral vessels cannulated in readiness for cardiopulmonary bypass prior to induction of general anesthesia. Those with less than $50 \%$ obstruction should have the femoral area prepared and draped for cannulation, should the need arise. While clearly not an option in a parturient, this recommendation highlights the need for meticulous planning should "plan A" turn into "plan B". Thorough preparation includes ensuring the availability and function of a fibreoptic bronchoscope, a rigid bronchoscope, and a variety of sizes and types of endotracheal tubes. Consideration should be given to ensure the availability of a second anesthesiologist and an otorhinolaryngologist or thoracic surgeon in case things deteriorate. Planning should continue into the postoperative period and following intrathecal morphine the patient should go to a monitored location, either an intensive care or step-down unit.

This patient was anemic at the time of presentation. Fluid administration was judiciously limited to $1500 \mathrm{cc}$. In the presence of potential aortocaval compression and SVC obstruction, a pregnant woman may require two $i v$ lines, one in each of an upper and lower extremity. If SVC obstruction is pronounced, an oxygen saturation monitor gives more useful information on a toe than on a finger. By the alveolar air equation we know that an oxygen saturation monitor does not give useful information about the adequacy of ventilation in the presence of supplemental oxygen. An arterial line would have provided valuable information in the presence of blood loss or respiratory compromise, and its absence is this case is notable. With compromised venous return, hypovolemia from postpartum hemorrhage is poorly tolerated. Pharmacological agents used in the management of obstetric hemorrhage should be administered with extreme caution, as they could have exaggerated and potentially life-threatening responses in a patient with profound cardiopulmonary disturbances. Ergot alkaloids can cause hypertension and peripheral vasoconstriction, further compromising organ perfusion. 15-Methyl prostaglandin $\mathrm{F}_{2 \text {-alpha }}$ (carboprost, or Hemabate $\left.{ }^{\circledR}\right)$ has been associated with arterial oxygen desaturation, pulmonary edema, hypotension, hypertension and bronchospasm.

It is indeed fortunate that the perioperative management of this patient proceeded without incident. Apart from elevating the head of the bed during the surgical procedure and the administration of fentanyl for cough control, the management of this patient with an anterior mediastinal mass did not differ in any respect from the usual care given to a healthy patient for elective Cesarean section. The nature of anterior mediastinal masses and the importance of avoiding general anesthesia in patients with this condition highlight the need to anticipate and plan for every conceivable complication that might occur. The unprepared anesthesiologist risks a catastrophic outcome. I still believe in the "carefully titrated epidural".

\section{References}

1 Van Winter JT, Wilkowske MA, Shaw EG, Ogburn PL $J r$, Pritchard DJ. Lung cancer complicating pregnancy: case report and review of literature. Mayo Clin Proc 1995; 70: 384-7.

2 Martin WJ. Cesarean section in a pregnant patient with an anterior mediastinal mass and failed supradiaphragmatic irradiation. J Clin Anesth 1995; 7: 312-5.

3 Ferrari LR, Bedford RF. Anterior mediastinal mass in a pregnant patient: anesthetic management and considerations. J Clin Anesth 1989; 1: 460-3.

4 Zeldis SM. Dyspnea during pregnancy. Distinguishing cardiac from pulmonary causes. Clin Chest Med 1992; 13: 567-85.

5 Elkus R, Popovich JJr. Respiratory physiology in pregnancy. Clin Chest Med 1992; 13: 555-65.

6 Montella KR. Pulmonary pharmacology in pregnancy. Clin Chest Med 1992; 13: 587-95.

7 Boyle RK. Cough stress fractures in two obstetric patients: case report and pathophysiology. Int J Obset Anesth 1998; 7: 54-8.

8 Check JH, Nowroozi K, Barnea ER, Shaw KJ, Sauer $M V$. Successful delivery after age 50: a report of two cases as a result of oocyte donation. Obstet Gynecol 1993; 81: 835-6.

9 Gilbert WM, Nesbitt TS, Danielsen B. Childbearing beyond age 40: pregnancy outcome in 24,032 cases. Obstet Gynecol. 1999; 93: 9-14.

10 Pullerits J, Holzman R. Anaesthesia for patients with mediastinal masses. Can J Anaesth 1989; 36: 681-8. 
11 Benumof JL, Alfery DD Anesthesia for Thoracic Surgery. In: Miller DD (Ed.). Anesthesia, 5th ed. Philadelphia: Churchill Livingstone, 2000; 48: 1736-8.

12 Hawkins JL, Koonin LM, Palmer SK, Gibbs CP. Anesthesia-related deaths during obstetric delivery in the United States, 1979-1990. Anesthesiology 1997; 86: 277-84.

13 Rout C, Rocke DA. Spinal hypotension associated with cesarean section. Will preload ever work? Anesthesiology 1999; 91: 1565-7.

14 Chan YM, Ngai SW, Lao TT. Gastric adenocarcinoma presenting with persistent, mild gastrointestinal symptoms in pregnancy. A case report. J Reprod Med 1999; 44: 986-8.

15 Heffner JE, Sahn SA. Pleural disease in pregnancy. Clin Chest Med 1992; 13: 667-78.

16 Weinreb HJ. Demyelinating and neoplastic diseases in pregnancy. Neurol Clin 1994; 12: 509-26.

17 Petrek JA. Pregnancy-associated breast cancer. Semin Surg Oncol 1991; 7: 306-10.

18 Toppenberg KS, Hill DA, Miller DP. Safety of radiographic imaging during pregnancy. Am Fam Phys 1999; 59: 1813-8.

19 American College of Obstetricians and Gynecologists, Committee on Obstetric Practice. Guidelines for diagnostic imaging during pregnancy. ACOG Committee opinion no. 158. Washington, DC. ACOG, 1995.

20 Reisner LS, Lin D. Anesthesia for cesarean section. In: Chestnut DH (Ed.). Obstetric Anesthesia: Principles and Practice, 2nd ed. St. Louis: Mosby, 1999: 465.

21 Patel M, Samsoon G, Swami A, Morgan B. Posture and the spread of hyperbaric bupivacaine in parturients using the combined spinal epidural technique. Can J Anaesth 1993; 40: 943-6.

22 Robson SC, Boys RJ, Rodeck C, Morgan B. Maternal and fetal haemodynamic effects of spinal and extradural anaesthesia for elective caesarean section. Br J Anaesth 1992; 68: 54-9.

23 Rout CC, Rocke DA, Gouws E. Leg elevation and wrapping in the prevention of hypotension following spinal anaesthesia for elective caesarean section. Anaesthesia 1993; 48: 304-8.

24 Shamberger RC. Preanesthetic evaluation of children with anterior mediastinal masses. Semin Pediatr Surg 1999; 8: 61-8.

25 Goh MH, Liu XY, Goh YS. Anterior mediastinal masses: an anaesthetic challenge. Anaesthesia 1999; 54: 670-82. 